\title{
CFTR, AN ION CHANNEL EVOLVED FROM AN ABC
}

TRANSPORTER

\section{Affiliation}

Paola Vergani ${ }^{1}$, David C. Gadsby ${ }^{2}$, László Csanády ${ }^{3}$

${ }^{1}$ University College London

London

UK

${ }^{2}$ Rockefeller University

New York, NY

USA

${ }^{3}$ Semmelweis University

Budapest

Hungary

\section{Introduction}

CFTR (cystic fibrosis transmembrane conductance regulator) is an integral membrane protein that functions as an epithelial $\mathrm{Cl}^{-}$channel, which is rendered defective by inherited gene mutations in patients with cystic fibrosis (Riordan et al. 1989). Its function underlies fluid secretion in the airways, sweat ducts, pancreatic duct, and vas deferens, and it mediates the excessive intestinal water loss in secretory diarrhoeas 
(Verkman and Galietta 2009). CFTR belongs to the large ABC (ATP-binding cassette) transporter superfamily, of which most members are ATP-hydrolyzing pumps. CFTR is the sole $\mathrm{ABC}$ protein known to function as an ion channel. Despite this difference in function, the gates of CFTR channels are opened and closed by interactions with ATP similar to those that in other $\mathrm{ABC}$ proteins result in energetically uphill substrate transport.

\section{CFTR is an ion channel}

A CFTR channel in its open conformation provides a pathway for $\mathrm{Cl}^{-}$ions (or other small anions, such as bicarbonate) to cross the cell membrane, down the gradient of their electrochemical potential. Because the ions moving through the channel carry an electrical current across the membrane, the patch-clamp recording technique (Gibb 1995) can be employed to study CFTR function. Once a saline-filled glass pipette connected to an electrode is sealed tightly against a small patch of membrane, the gating of any CFTR channels present in the patch can be accurately monitored by observing how the current flowing through the membrane patch changes with time. If the patch contains few channels, individual events reflecting opening and closing of single-channel gates can be detected as abrupt changes in the current level (Fig. 1A, black trace, recorded in a patch with one active CFTR channel).

For quantitative analysis of such current records, gating is treated as a stochastic process described by a kinetic scheme (which includes the states the channel can visit, how those states are connected, and rates of transitions between the states; e.g. Fig. 1B inset, Hidden Markov modeling). The first task for the analysis is collection of all dwell 
times in the closed state (represented by green bars in Fig 1A), and of all dwell times in the open state (red bars). In general, dwell times in a particular state provide information about the rates of leaving that state. Simple calculation of average dwell times can yield useful information, but a more powerful approach is analysis of distributions of dwell times (e.g., simulated open dwell-time distribution shown in Fig. 1B, Data analysis, Single Molecule Kinetic methods; Colquhoun and Hawkes 1995; Colquhoun and Sigworth 1995). In this analysis, the time axis is subdivided into bins and the ordinate reports the number of dwell times observed to have a duration that falls within the period encompassed by each bin. Dwell times in a single conformational state are exponentially distributed, which means that the bins containing brief durations have the highest event count, and the distribution decays monotonically. This is because the probability of leaving a state is the same for each time interval spent in that state, so that the chance for a channel to survive in a given state declines with time. Fitting of dwell-time distributions using a statistical procedure called maximum likelihood (ML) can give rate estimates for the underlying kinetic scheme. For example, in Fig. 1B the red curve superimposed on the depicted simulated open dwell-time distribution data is the theoretical open dwelltime distribution predicted for a $\mathrm{C} \leftrightarrow \mathrm{O}$ model that has a closing rate, $\mathrm{k}_{\mathrm{OC}}$, of $\sim 4 \mathrm{~s}^{-1}$ (Fig. 1B, inset; this was the model used to simulate the data). According to the ML criterion, this curve is the "best fit" to the observed distribution. Thus, given a kinetic scheme, ML fitting finds values for the rates (e.g. rate $\mathrm{k}_{\mathrm{OC}}$ for the open dwell-time distribution in Fig.1B) such that the likelihood of obtaining the observed distribution is maximized.

\section{CFTR_Fig1.tif}


Figure 1A. Single CFTR channel current trace (black), in which upward deflections correspond to channel-opening events that allow $\mathrm{Cl}^{-}$ions to flow across the patch. [Note 1: in this case, anions flow from cytoplasmic to extracellular side of the membrane, constituting an inward current; but, for consistency between figures, in this review channel openings are plotted as upward deflections in all figures, regardless of the actual direction of ion flow determined in each case by the $\mathrm{Cl}^{-}$electrochemical gradient.] The current flowing through open channels corresponds to $\sim 3$ million $\mathrm{Cl}^{-}$ions per second. [Note 2: although not evident in the traces shown in this review, at a higher time resolution CFTR can be seen to open into "bursts" of openings: clusters of openings separated by short-lived, "flickery" closures. Duration and frequency of flickery closures are not nucleotide-dependent. Rather, NBD dimer formation and dissociation drive initiation and termination of bursts; here, for simplicity, we use the terms opening and closing to indicate entry and exit from bursts.] Red and green bars illustrate length of individual dwell-times in open and closed states, respectively.

Figure 1B. Example of an open dwell-time distribution (yellow histogram), and ML fit (red line), for a current trace simulated using the simple kinetic scheme in the inset, with $\mathrm{koC}_{\mathrm{OC}}=4 \mathrm{~s}^{-1}$.

Two main factors influence the gate in a CFTR channel: they are phosphorylation, principally by (cAMP-dependent) protein kinase A (PKA), and interaction with ATP (Fig. 2; Gadsby et al. 2006). Phosphorylation is a prerequisite for CFTR channel gating in 
response to binding and hydrolysis of ATP. By adding PKA and ATP to the cytosolic side of the patch, channels become phosphorylated; after that the current starts fluctuating in a stepwise fashion. This pattern reflects fluctuations in the number of channels that are open at any point in time. Once the channels have been phosphorylated, ATP is sufficient and necessary for channel gating: adding ATP can activate them, but after washing the ATP away no further openings occur (Fig. 2).

\section{CFTR_Fig2.tif}

Figure 2. Current trace from patch containing at least 3 CFTR channels; the number simultaneously open is indicated at the right. Solid lines below the trace signify the presence at the cytosolic face of the patch of the specified reagent: red line, 5 mM ATP; blue line, catalytic subunit of PKA.

At the molecular level, ATP interacts with CFTR's two cytosolic nucleotide binding domains (NBDs), NBD1 in the N-terminal half of CFTR, and NBD2 near the C terminus, whereas phosphorylation occurs at several serines within the cytosolic "regulatory" (R) domain, a region unique to CFTR and absent from other ABC proteins. On the other hand, the permeation pathway through which $\mathrm{Cl}^{-}$ions cross the membrane is constructed by the two transmembrane domains (TMDs, Fig. 3A).

\section{CFTR's NBDs and their role in channel gating}

High resolution crystal structures have been determined for many NBDs, including CFTR's NBD1 and a modified NBD2. In all ABC proteins, nucleotide interacts 
directly with conserved sequences of residues (Walker motifs) in the "head" of an NBD (Fig. 3B). But the conserved ABC signature (LSGGQ-like) sequence occurs in the helical "tail" of the NBD, quite distant from where the nucleotide is found bound in monomeric crystals. However, NBDs can interact to form head-to-tail dimers, with an ATP molecule buried within each of the two composite nucleotide-binding sites created at the dimer interface (Fig. 3B). The ATP acts as "molecular glue", with the two ATP molecules, and in particular their $\gamma$-phosphates, providing molecular contacts to both sides of the composite binding sites and thereby making important contributions to dimer stability.

\section{CFTR_Fig3.tif}

Figure 3. A: CFTR topology including TMDs (grey), NBD1 (green), NBD2 (blue) and $\mathrm{R}$ domain (white). Intracellular linking loops are shown in magenta. B: crystal structure of an NBD homodimer, from a bacterial $A B C$ transporter. C: schematic representation of a head-to-tail NBD dimer.

NBD1 and NBD2 form an intramolecular heterodimer with two distinctly different composite binding sites at the interface, referred to as composite site 1 and composite site 2, comprising Walker motifs in the head of NBD1 and head of NBD2, respectively (Heteromeric versus homomeric association).

Quantitative analysis of how ATP affects CFTR channel gating has been carried out by exposing patches to different test [ATP] and monitoring how gating parameters are altered (Fig. 4). Closing rate (reciprocal of the mean open dwell time) shows no clear dependence on [ATP] over a three orders of magnitude concentration range (Fig. 4C). In 
contrast, over the same [ATP] range, the opening rate (reciprocal of the mean closed dwell time) first increases and then saturates (Fig.4B). The data are described by Michaelis-Menten kinetics, with apparent ATP affinity $\sim 50 \mu \mathrm{M}$ (or the Hill equation, with Hill coefficient of 1). Therefore, at low [ATP] CFTR channel opening is rate-limited by an ATP binding step, which means that ATP binding must occur on closed channels, and precedes channel opening; the Hill coefficient of 1 is consistent with a single binding step controlling opening.

\section{CFTR_Fig4.tif}

Figure 4. [ATP]-dependence of CFTR gating. A: current trace from patch containing multiple CFTR channels, exposed to a test concentration of $50 \mu \mathrm{M}$ ATP, in between control exposures to $5 \mathrm{mM}$ ATP. Red and green bars highlight duration of dwell times at conductance levels 1 (one open channel) and zero (no open channel). B, C: [ATP]-dependence of opening rate and closing rate, respectively.

ABC proteins do not merely bind ATP, they also catalyse ATP hydrolysis (ATPase: Overview). Native, wild-type (WT) CFTR, too, is an active ATPase, with a measured turnover rate on the order of $1 \mathrm{ATP} \mathrm{s}^{-1}$ (Li et al. 1996). How this hydrolysis affects gating is most readily observed by studying channel closing rates in patches containing large numbers (hundreds, or even thousands) of CFTR channels (e.g. records in Fig. 5 - compare current scale to those in Figs. 1, 2; Analysis of macroscopic currents). In such recordings, individual opening and closing events are lost in noise. Upon removal 
of ATP, the opening rate falls to almost 0 (see Figs. 2, 4B), so the time course of current decline reflects only the channel closing rate. WT channels close with a time constant below 1s (Fig. 5A). Mutation of a crucial catalytic lysine, K1250, in the Walker A motif of composite site 2, abolishes ATPase activity of purified, reconstituted CFTR (Li et al. 1996), and K1250A CFTR channels close extremely slowly (time constant tens of seconds, Fig. 5B). Mutation of other catalytic residues in composite site 2 (e.g. E1371, the "catalytic glutamate", and D1370, see below) also slows channel closure. These observations suggest that hydrolysis at site 2 is required for normal, fast channel closure.

\section{CFTR_Fig5.tif}

Figure 5. Current recordings from patches containing hundreds of WT (A) or mutant K1250A (B) CFTR channels. Macroscopic current decay upon abrupt removal of ATP monitors channel closing rate (superimposed blue lines are single exponential fits).

In contrast, CFTR channels carrying a mutation at the equivalent conserved lysine, K464, in the Walker A motif of composite site 1, appear to close normally and display average open dwell times not different from those of WT channels. In fact, biochemical experiments suggest that, even in WT CFTR, ATP binds tightly in composite site 1 but remains there for minutes without being hydrolyzed. This lack of activity is accounted for by several non-canonical substitutions of otherwise conserved key residues on both sides of the NBD1-NBD2 heterodimer interface in composite site 1. Although gating kinetics of K464A mutant channels look very similar to those of WT, 
biochemical measurements show that the ATPase rate of K464A is only one tenth that of WT (Ramjeesingh et al. 1999). This will be considered again later (see Evidence for a non-equilibrium gating scheme for $\mathrm{CFTR}$ ). Other $\mathrm{ABC}$ proteins (e.g., multidrugresistance related proteins, sulfonylurea receptors, transporter associated with antigen processing) share with CFTR the presence of a non-canonical interfacial ATP binding site, so that only one of their two composite sites catalyzes ATP hydrolysis at a measurable rate.

The inference from findings like these is that a scheme that relates ATP interactions with the NBDs to channel gating must include the following features. ATP binds tightly to the Walker motifs at the NBD1 head (in composite site 1) where it remains, unhydrolyzed, for several gating cycles. Then, after ATP binds to the Walker motifs at the NBD2 head of composite site 2, i.e., once ATP is bound at both sites, the channel can open. The open state of the channel, with 2 ATP molecules bound, is very stable, and rapid closure can occur only after the ATP at composite site 2 is hydrolyzed. Fig. 6A shows a working hypothesis for such a CFTR gating scheme, in terms of channel closed $\left(\mathrm{C}_{1}, \mathrm{C}_{2}\right)$ and channel open $\left(\mathrm{O}_{1}, \mathrm{O}_{2}\right)$ conformations (only the nucleotide bound and hydrolyzed - at site 2 is indicated; ATP bound at site 1, assumed present in all 4 states, is omitted for simplicity). A structural interpretation of this scheme can be made in the light of structural knowledge recently obtained for other ABC proteins (Locher 2009): conformational changes in the TMDs that open and close the $\mathrm{Cl}^{-}$ion pathway could be coupled to the ATPase cycle via formation and dissociation of the NBD1-NBD2 dimer (Fig. 6B). Formation of an NBD1-NBD2 dimer after binding of both ATPs could be coupled to opening of the channel pore. Then hydrolysis of the ATP at site 2 would 
destabilize the NBD dimer, triggering fast channel closure. So, while ATP at site 1 remains bound, without being hydrolyzed, for many gating cycles, the ATPase cycle at site 2 is coupled to opening and closing of the $\mathrm{Cl}^{-}$permeation pathway. This interpretation is supported by experimental evidence, obtained using double mutant cycle analysis, that opening of the CFTR pore corresponds to closure of the NBD1-NBD2 dimer interface gap at composite site 2 (Gadsby et al. 2006).

\section{CFTR_Fig6.tif}

Figure 6. Working hypothesis linking ATPase and gating cycles of CFTR. A: Proposed gating scheme relating nucleotide present at site 2 to channel state (closed states, $\mathrm{C}_{1}$ and $\mathrm{C}_{2}$; open states, $\mathrm{O}_{1}$ and $\mathrm{O}_{2}$ ). B: Structural interpretation of gating scheme in A, colour coding as in Figure 3 . The small orange balls represent $\mathrm{Cl}^{-}$ions flowing through CFTR's transmembrane pore.

\section{Phosphorylation of the $\mathrm{R}$ domain relieves gating inhibition}

R-domain phosphorylation is required for CFTR channel activation, and can be considered the major regulatory mechanism in vivo because the millimolar range of [ATP] in cells is enough to fully activate channel gating (Fig. 4). Studies on full-length CFTR in intact cells, and on isolated R-domain peptides in vitro, have identified at least ten R-domain serines that become phosphorylated by PKA, most of them occurring within dibasic PKA recognition consensus sequences $(\mathrm{R} / \mathrm{K}, \mathrm{R} / \mathrm{K}, \mathrm{X}, \underline{\mathrm{S}})$. This large number of target serines allows for a graded functional response, and CFTR channel activity 
increases in rough proportion to phosphorylation stoichiometry(reviewed in Gadsby et al. 2006).

Either of two kinds of regulatory mechanisms could account for the findings. The unphosphorylated $\mathrm{R}$ domain could inhibit channels that would be active in its absence, or the phosphorylated $\mathrm{R}$ domain could stimulate channels that would be otherwise inactive. Experiments show that excision of the R domain results in constitutive, phosphorylationindependent, channel activity; indeed, cutting CFTR in half and coexpressing the two segments TMD1-NBD1 and TMD2-NBD2 yields "cut $\triangle \mathrm{R}$ " CFTR channels whose ATPdependent gating in the absence of PKA phosphorylation is comparable to that of phosphorylated WT CFTR. Because the stimulation of WT channels by PKA corresponds to a $>100$-fold increase in open probability $\left(\mathrm{P}_{\mathrm{o}}\right)$, while the constitutive $\mathrm{P}_{\mathrm{o}}$ of cut $\Delta \mathrm{R}$ is about half that of fully phosphorylated WT, the dominant role of the R domain appears to be inhibition of channel activity when the R domain is dephosphorylated. A small, $<2$ fold, stimulation by the phosphorylated R domain has also been proposed(reviewed in Gadsby et al. 2006).

How phosphorylation influences R-domain structure and its interactions with the rest of CFTR remains unclear. The $\mathrm{R}$ domain lacks sequence homology to any known protein, and CD spectral analysis and NMR studies of isolated R-domain peptide indicate the absence of stable 3-dimensional structure (Structurally disordered Proteins). Nevertheless, phosphorylation of R-domain peptide by PKA diminished the -helical contribution to its $\mathrm{CD}$ spectrum. Correspondingly, NMR measurements revealed stretches of dephosphorylated R-domain peptide, near target serines, with distinct helical propensity and a tendency to interact with isolated NBD1 protein; and both 
characteristics were weakened by phosphorylation. But whether such interactions between the R domain and NBD1 occur in intact CFTR and, if so, whether they are modulated by phosphorylation is not known (Gadsby et al. 2006; Baker et al. 2007).

Phosphorylation is unlikely to exert its effect through mere accumulation of negative charge. Although mutation of 8 consensus serines to negatively charged aspartates or glutamates results in some constitutive, PKA-independent, channel activity, this constitutive $\mathrm{P}_{\mathrm{o}}$ is very low compared to that of fully phosphorylated WT, suggesting that negative charge per se is a poor mimic of phosphorylation. Also, phosphorylation of serines 737 and 768 decreases $P_{0}$, which again argues against a simple effect of charge accumulation.

Similarly, the graded increase of channel $\mathrm{P}_{\mathrm{o}}$ with phosphorylation stoichiometry is hard to reconcile with initial suggestions that the unphosphorylated $\mathrm{R}$ domain might physically obstruct the pore; and phosphorylation has no major effect on ATP binding affinity of the NBDs. Because phosphatase-mediated dephosphorylation essentially abolishes ATP hydrolysis of purified CFTR protein (Li et al. 1996), the catalytic cycle appears to be stalled in unphosphorylated CFTR, rather than uncoupled from gating. However, because catalytic and pore-gating cycles appear to be strictly coupled (Csanády et al., 2010), the "brake" could arrest either the NBDs (e.g., by preventing NBD dimer formation, see below) or the TMDs (e.g., by preventing conformational changes associated with pore opening) - just as a see-saw can be stopped by immobilizing either end. Interestingly, two non-conserved segments in NBD1, both containing phosphorylatable serines, had been suggested to prevent NBD dimerization in their nonphosphorylated states, but deletion of neither segment affected strict phosphorylation 
dependence of gating. Instead, mounting evidence suggests that the "brake" acts on the TMDs rather than on the NBDs. First, simple severing of covalent linkage between the R domain and TMD2 (between residues 835 and 837) disrupts strict phosphorylationdependence of gating, yielding channels with $\sim 20 \%$ of maximal activity in the absence of phosphorylation. More importantly, the completely ATP-independent gating of truncated CFTR channels, lacking the entire NBD2 domain, remains strictly dependent on phosphorylation. This suggests that the R domain directly interacts with the intracellular linking loops of the TMDs, consistent with conclusions from low-resolution cryo-EM imaging. By altering the specific pattern of interactions between the $\mathrm{R}$ domain and the intracellular linking loops and/or CFTR's $\mathrm{N}$ terminal tail, phosphorylation likely lowers the energetic cost of the TMD conformational change associated with pore opening. Once the "weight" on the TMD-end of the see-saw has been thus diminished, the energy released during ATP-dependent NBD dimer formation, in WT CFTR, becomes sufficiently "heavy" to flip the see-saw from an open-dimer/closed-pore conformation into a tight-dimer/open-pore state (Gadsby et al. 2006; Zhang et al. 2009; Wang 2010; Wang et al. 2010).

Besides PKA, other kinases can phosphorylate the R domain, some targeting the same serines as PKA, others distinct sets of residues. Phosphorylation of serine 686 by protein kinase $\mathrm{C}(\mathrm{PKC})$ seems to play a permissive role: PKA-dependent CFTR activation requires prior phosphorylation of serine 686 by PKC. Recently, the "inhibitory" serine 768 (see above) was shown to be a substrate of AMP-kinase (AMPK), which explains the suppressive effect of AMPK activity on whole-cell CFTR currents (Hallows et al. 2000). Still unclear are functional and physiological consequences of 
CFTR phosphorylation by cyclic GMP-dependent protein kinase (PKG), and calcium/calmodulin-dependent protein kinase I (CaM kinase I)(Gadsby et al. 2006; King et al. 2009; Kongsuphol et al. 2009).

\section{Evidence for a non-equilibrium gating scheme for CFTR}

The cyclic kinetic scheme underlying CFTR gating (Fig. 6) is not yet universally accepted. Some researchers believe a simple equilibrium kinetic scheme best describes CFTR gating (Aleksandrov et al. 2009). In an equilibrium scheme (within blue dotted line, Fig. 7 top), closing and opening occur along the same pathway (i.e. closing is simply reversal of the opening transition). Such a scheme predicts a monotonically decaying open dwell-time distribution (as in Fig. 1B). In the non-equilibrium, cyclic, gating scheme in Fig 6A, for most opening events, closing occurs through a different pathway than opening. After opening (to state $\mathrm{O}_{1} \cdot \mathrm{ATP}$ ), because the non-hydrolytic closing pathway $\left(\mathrm{O}_{1} \cdot \mathrm{ATP}\right.$ to $\left.\mathrm{C}_{1} \cdot \mathrm{ATP}\right)$ is slow, the channel usually proceeds to a second open state. It transits through these two sequential open states, and only then it closes. This causes a rarity of brief open events. Therefore the non-equilibrium scheme predicts a peaked open dwell-time distribution with a rising phase, reflecting low event counts in the bins corresponding to shortest open dwell times.

For a given observed dwell-time distribution, two alternative ML fits can be obtained: one assuming the equilibrium scheme, and another assuming the nonequilibrium scheme. The log-likelihood score (LL) quantitatively describes the "goodness" of a fit. A LLEQULIBRIUM, assuming the equilibrium scheme, can be calculated as well as a $L L_{\text {NON-EQUILIBRIUM, }}$ assuming the non-equilibrium scheme. If the number of 
free parameters is the same in the two schemes being compared, the model that yields a higher LL value is the one that best describes the data. But if the number of free parameters is different, the choice is not as simple. More free parameters often allow a better fit, even if the extra parameters are not really required.

In cases in which models have different numbers of free parameters, can the significance of a better fit be quantified? The answer is yes, if the model with fewer parameters is a submodel of the more complex one. Since the equilibrium scheme is a submodel of the cyclic scheme (Fig. 7 top) the significance of a better fit can be quantified. This is done using the log-likelihood ratio $(\Delta \mathrm{LL})$, which quantifies the improvement in fit: $\Delta \mathrm{LL}=\mathrm{LL}_{\text {NON-EQUILIBRIUM }}-\mathrm{LL}_{\text {EQUILIBRIUM. }}$. The distribution of expected $\Delta \mathrm{LL}$ can be calculated assuming the simple model is true, and the observed value of $\Delta \mathrm{LL}\left(\Delta \mathrm{LL}_{\mathrm{obs}}\right)$ can be used to calculate a $P$ value: the probability of observing the $\Delta \mathrm{LL}_{\mathrm{obs}}$ assuming the simple scheme is true (Csanády 2006).

Fig. 7A shows the WT open dwell-time distribution (yellow histogram bars). The ML fit assuming the equilibrium model is the blue dotted line, and the ML fit assuming the non-equilibrium model is the red line. Using $\Delta \mathrm{LL}_{\mathrm{obs}}$ the alternative gating schemes can be ranked. The cyclic scheme provides a far better fit to the observed data: the $P$ value (the probability of obtaining the observed distribution, with several of the leftmost bins having relatively low counts, assuming that the underlying scheme is the equilibrium one) is very low, $2 \cdot 10^{-9}$. Thus the data are consistent with a kinetic scheme in which rate $\mathrm{k}_{-1}$ (non-hydrolytic closing) is negligibly small (i.e. the ATP-bound open state is very stable) so that for most openings the channel must hydrolyze ATP before it can close. It 
must go through two sequential steps before closing, resulting in a rarity of very brief open events. So, for WT CFTR, gating and ATP hydrolysis are strictly coupled.

\section{CFTR_Fig7.tif}

Figure 7. Open dwell-time distributions (yellow histograms) for WT (A), D1370N mutant (B) and K464A mutant (C) CFTR channels, and ML fits assuming either the equilibrium scheme (top left scheme, dotted blue lines) or the non-equilibrium scheme (top right scheme, solid red lines). For each distribution $P$ values are shown, which describe significance of improvement of fit by using the nonequilibrium scheme.

If a mutation is introduced into CFTR's catalytic composite site 2, at the conserved Walker B motif aspartate (D1370N, a mutation that in other ABC proteins abolishes hydrolysis), the open dwell-time distribution becomes monotonically decaying (Fig. 7B). There is no significant improvement using the more complex model, and the observed data are consistent with the hydrolytic rate $\mathrm{k}_{1}$ falling to zero. Thus, for this mutant, the cyclic scheme is reduced to the equilibrium scheme.

Introduction of the mutation K464A, at the crucial Walker A motif catalytic lysine in CFTR's non-canonical composite site 1, the open dwell-time distribution decays monotonically (Fig. 7C). But very short open dwell times are somewhat less frequent than would be expected assuming an equilibrium scheme (compare leftmost bins of yellow histogram bars to blue dotted line in Fig. 7C). If the $\mathrm{k}_{-1}$ rate is not constrained, the fit can be significantly improved by using the cyclic model. The $P$ value (the probability 
of obtaining this rarity of brief events by chance, assuming the equilibrium model is true), is 0.027 . The data are more consistent with a kinetic scheme in which the non-hydrolytic closing rate, $\mathrm{k}_{-1}$, is increased (possibly linked to a destabilization of the NBD1-NBD2 dimer), and the hydrolytic rate $\mathrm{k}_{1}$ is reduced. So for K464A mutant CFTR channels, in 4 out of 5 openings, closing occurs through reversal of the opening transition: in this mutant coupling between the ATPase and gating cycles is partially lost.

Using the rates estimated from ML fitting to dwell-time distributions for the WT and the two mutants, the ratio $\mathrm{k}_{1} /\left(\mathrm{k}_{1}+\mathrm{k}_{-1}\right)$, which describes the proportion of channel openings that are coupled to ATP hydrolysis, can be quantified. For WT, the ratio suggests $>95 \%$ coupling. For K464A, the ratio suggests $\sim 20 \%$ coupling (reduced $\mathrm{k}_{1}$, increased $\mathrm{k}_{-1}$ ); and, because the overall time taken for the whole gating cycle is also twofold longer, the result is in very good agreement with the reported $\sim 10$-fold slower ATPase turnover (see above; Ramjeesingh et al. 1999). For D1370N, coupling is completely abolished, as expected for a mutation that drastically impairs hydrolysis (Csanády et al. 2010).

\section{$\mathrm{Cl}^{-}$permeation pathway}

Although the gating mechanism of CFTR has been steadily coming into focus, until recently very little was known on the location of the channel gate(s) and on the identity of residues that line the hydrophilic anion permeation pathway through CFTR. Effects of substitutions (cysteines and/or alanines) at several sites (including R334, K335, F337, T338 in transmembrane helix 6, TM6, and K95, Q98 and P99 in TM1) on singlechannel current and channel permeation properties, suggest that these positions might line 
the channel pore or at least play a role in maintaining pore structure in the open channel state (Ge et al. 2004; Bai et al. 2011). Recent development of a functional "cys-less" CFTR (Mense et al. 2006), with all eighteen endogenous cysteines substituted by alternative amino acids, has facilitated systematic investigation of pore structure. After introduction of a unique target cysteine at a chosen location in cys-less CFTR, its accessibility to, and consequences of modification by, thiol-specific channel-permeant or -impermeant reagents can be monitored (Substituted Cysteine Accessibility Method, SCAM). As a result, structures that delineate CFTR's pore are now beginning to be identified (Linsdell 2006; Wang et al. 2011; Norimatsu et al. 2012).

Cysteines introduced at I331, L333, R334, K335, I336, T338 can be modified by thiol-specific reagents added to the extracellular solution, and I331C, L333C, K335C are modified more slowly in the open channel state, indicating changes in the accessibility of these residues during channel gating (Beck et al. 2008, Fig. 8A-C, orange). Moreover, with extracellularly applied thiol-reactive probes, cysteines at positions intracellular to F337 (e.g., T339, I340, S342, Fig. 8A-C, red) react only with small reagents capable of permeating the channel pore (Alexander et al. 2009). Of interest, reactivity of V345C to thiol-specific reagents applied at the intracellular side of the membrane seemed independent of channel gating state, whereas I344C reacted only in the channel open state, interpreted as suggesting that some accessibility "barrier" between positions 345 and 344 might be removed during channel opening (El Hiani and Linsdell 2010). Also, alterations of $\mathrm{P}_{\mathrm{o}}$ caused by covalent modification of cysteines introduced along TM6, were similarly interpreted to suggest that part of this helix might contribute to the channel gate and move relative to other TMs during gating (Bai et al. 2010). On TM1, K95C was 
modified regardless of channel state, while Q98C was accessible only in the open state, implying that K95/Q98 on TM1 and I344/V345 on TM6 might lie at a similar depth along the pore axis, a suggestion supported by disulfide bridge formation between $\mathrm{K} 95 \mathrm{C}$ or Q98C and I344C, associated with marked reduction in current (Wang et al. 2011, Fig. 8D). Very recent SCAM scans along TM12 suggest that it too likely makes significant contributions to the surface lining the pore. Open-state-dependent accessibility to reagents applied from the cytosolic side for N1138C and M1140C, but not S1141, again might imply location of a gating "barrier" between the latter two residues (Qian et al. 2011, Fig. 8D).

Despite these steps forward, a clear picture of how an anion selective pathway is formed through CFTR in the open-channel conformation, but is interrupted in the closedchannel state, has yet to emerge. Presently available crystal structures of homologous $\mathrm{ABC}$ exporter proteins group into "outward facing" conformations with closely juxtaposed NBDs and with TMDs forming a cavity open to the extracellular solution, and "inward facing" conformations with NBDs very distant from each other and with a wide intracellular vestibule. Short "coupling helices" (magenta; Fig. 8A,B) at the intracellular linking loop ends furthest from the membrane, but positioned parallel to the membrane plane, contact the surfaces of the NBDs in a "domain-swap" arrangement and are believed to transmit the conformational signal generated by NBD dimerization to the TMDs. Based on evidence that CFTR channel opening corresponds to the formation of a tight NBD dimer (Gadsby et al. 2006; Mense et al. 2006), the expectation is that CFTR channel opening also corresponds to the flipping of TMDs from "inward facing" to "outward facing". However, in the latter conformation, helices corresponding to CFTR's 
TM1, TM12 and TM6 are modelled to be very distant (in homology models these helices are separated by between $14 \AA$ and $18 \AA$, at the level of the putative "barriers", Fig. 8D), making it difficult to envisage how in such a conformation they could form a pathway selective for anions. Therefore the limited structures available so far, and the limitations of homology models, combine to leave unanswered the question of precisely how these TMs contribute to the anion selective pathway (but see Norimatsu et al. 2012).

\section{CFTR_Fig8.tif}

Figure 8. Anion permeation pathway. Homology model of CFTR, built on the "outward facing" crystal structure of a bacterial homolog (Sav1866, Locher 2009). Only selected residues mentioned in text are shown. In TM1: K95 in dark green and Q98 in light green. In TM6: I331, L333, K335 in orange; R334, T338 in yellow; T339 and F342 in red; I344 in pink; V345 in purple. In TM12: N1138 and M1140 in light blue; S1141 in dark blue. A, B: View of CFTR model in membrane (light grey), with and without NBDs. Domains are colour-coded as in Figs. 3 and 6; R domain, not present in homologous crystal structures, is omitted. C: Extracellular view of outer mouth of pore. D: Outermost structural elements have been removed from $\mathrm{C}$, to show transmembrane helices at the level of suggested permeation "barrier": residues coloured with lighter hues are accessible to intracellular reagents only in the open-channel state.

\section{Summary}


CFTR, encoded by the gene which is mutated in cystic fibrosis patients, belongs to the superfamily of $\mathrm{ABC}$ transporters but is a quite unusual family member. First, unlike all other members examined so far, it functions as an ion channel: when open, millions of $\mathrm{Cl}^{-}$ions flow through it every second, down their electrochemical gradient. CFTR seems to work as a broken pump. Instead of securely occluding its transported "substrate" in a binding site which never has simultaneous access to both faces of the membrane, one of the two pump gates is always open, allowing some conformations within the CFTR catalytic cycle to form a continuous aqueous pathway from extracellular solution to cytosol (Gadsby 2009). A first picture of the molecular structures lining this permeation pathway is beginning to emerge. Second, unlike other ABC transporters it requires phosphorylation of several serines in its unique $\mathrm{R}$ domain before it becomes active. But CFTR is not only unique as an ABC protein, it is also very unusual as an ion channel. What is known about the molecular mechanism underlying its ATP-dependent gating suggests that gating is strictly coupled to an ATP hydrolysis cycle. This behavior is typical of active transporters. But is it just a vestige of CFTR's evolution from its ABC transporter ancestors? Or could some of the many proteins that interact with CFTR slow its ATPase activity, thereby prolonging channel-open dwell times and so providing a sensitive means of regulating channel activity, analogous to modulation of the GTPase activity of G proteins? Or could CFTR still be an active transporter of some as yet unknown substrate, and the concomitant electrodiffusive flow of $\mathrm{Cl}^{-}$be a paraphenomenon -- albeit a physiologically vital one -- of that transport process? 


\title{
Hyperlinked
}

\author{
$\mathrm{ABC}$ transporters \\ Analysis of macroscopic currents \\ ATPase: Overview \\ Data analysis \\ Domain swapping \\ Double mutant cycle analysis \\ Heteromeric versus homomeric association \\ Hidden Markov modeling \\ KATP and the sulphonylurea receptor \\ Membrane proteins \\ Patch clamp recording of single channel activity - acquisition and analysis \\ Phosphorylation \\ Single Molecule Kinetic methods \\ Structurally disordered Proteins \\ Substituted Cysteine Accessibility Method
}

\section{References}

Aleksandrov AA, Cui L and Riordan JR (2009). Relationship between nucleotide binding and ion channel gating in cystic fibrosis transmembrane conductance regulator. J Physiol 587 2875-2886 
Alexander C, et al. (2009). Cystic Fibrosis Transmembrane Conductance Regulator:

Using Differential Reactivity toward Channel-Permeant and Channel-Impermeant Thiol-Reactive Probes To Test a Molecular Model for the Pore. Biochemistry 48: $10078-10088$

Bai Y, Li M and Hwang T-C (2010). Dual roles of the sixth transmembrane segment of the CFTR chloride channel in gating and permeation. J Gen Physiol 136: 293-309

Bai Y, Li M and Hwang T-C (2011). Structural basis for the channel function of a degraded ABC transporter, CFTR (ABCC7). J Gen Physiol 138: 495-507

Baker JMR, et al. (2007). CFTR regulatory region interacts with NBD1 predominantly via multiple transient helices. Nat Struct Mol Biol. 14: 738-745

Beck EJ, Yang Y, Yaemsiri S and Raghuram V (2008). Conformational Changes in a Pore-lining Helix Coupled to Cystic Fibrosis Transmembrane Conductance Regulator Channel Gating. J Biol Chem 283: 4957-4966

Colquhoun D and Hawkes AG (1995). The Principles of Stochastic Interpretation of IonChannel Mechanisms. In Sackmann B and Neher E (Ed) Single Channel Recording. Plenum Press, New York, pp 397-479.

Colquhoun D and Sigworth FJ (1995). Fitting and Statistical Analysis of Single-Channel Records. In Sackmann B and Neher E (Ed) Single Channel Recording. Plenum Press, New York, pp 483-585.

Csanády L (2006). Statistical Evaluation of Ion-Channel Gating Models Based on Distributions of Log-Likelihood Ratios. Biophys. J. 90: 3523-3545 
Csanády L, Vergani P and Gadsby DC (2010). Strict coupling between CFTR's catalytic cycle and gating of its $\mathrm{Cl}^{-}$ion pore revealed by distributions of open channel burst durations. Proc Natl Acad Sci U S A 107: 1241-1246

El Hiani Y and Linsdell P (2010). Changes in Accessibility of Cytoplasmic Substances to the Pore Associated with Activation of the Cystic Fibrosis Transmembrane Conductance Regulator Chloride Channel. J Biol Chem 285: 32126-32140

Gadsby DC (2009). Ion channels versus ion pumps: the principal difference, in principle. Nat Rev Mol Cell Biol 10: 344-352

Gadsby DC, Vergani P and Csanády L (2006). The ABC protein turned chloride channel whose failure causes cystic fibrosis. Nature 440: 477-483

Ge N, Muise CN, Gong X and Linsdell P (2004). Direct Comparison of the Functional Roles Played by Different Transmembrane Regions in the Cystic Fibrosis Transmembrane Conductance Regulator Chloride Channel Pore. J. Biol. Chem. 279: 55283-55289

Gibb AJ (1995). Patch-clamp Recording. In Ashley RH (Ed) Ion Channels: a practical approach. Oxford University Press, Oxford, pp 1-42.

Hallows KR, Raghuram V, Kemp B, Witters LA and Foskett JK (2000). Inhibition of cystic fibrosis transmembrane conductance regulator by novel interaction with the metabolic sensor AMP-activated protein kinase. J Clin Invest. 105: 1711-1721

King JD, et al. (2009). AMP-activated protein kinase phosphorylation of the R domain inhibits PKA stimulation of CFTR. Am J Physiol Cell Physiol. 297: C94-C101 Kongsuphol P, et al. (2009). Mechanistic Insight into Control of CFTR by AMPK. J. Biol. Chem. 284: 5645-5653 
Li C, et al. (1996). ATPase activity of the cystic fibrosis transmembrane conductance regulator. Journal of Biological Chemistry 271: 28463-8

Linsdell P (2006). Mechanism of chloride permeation in the cystic fibrosis transmembrane conductance regulator chloride channel. Experimental Physiology 91: $123-129$

Locher KP (2009). Structure and mechanism of ATP-binding cassette transporters. Philos. Trans. R. Soc. Lond. B Biol. Sci. 364: 239-245

Mense M, et al. (2006). In vivo phosphorylation of CFTR promotes formation of a nucleotide-binding domain heterodimer. EMBO J. 25: 4728-4739

Norimatsu Y, et al. (2012) CFTR: A molecular model defines the architecture of the anion conduction path and locates a "bottleneck" in the pore. Biochemistry DOI: $10.1021 / \mathrm{bi} 201888 \mathrm{a}$

Qian F, El Hiani Y and Linsdell P (2011). Functional arrangement of the 12th transmembrane region in the CFTR chloride channel pore based on functional investigation of a cysteine-less CFTR variant. Pflugers Arch. 462: 559-571

Ramjeesingh M, et al. (1999). Walker mutations reveal loose relationship between catalytic and channel-gating activities of purified CFTR (cystic fibrosis transmembrane conductance regulator). Biochemistry 38: 1463-8

Riordan JR, et al. (1989). Identification of the cystic fibrosis gene: cloning and characterization of complementary DNA. Science 245: 1066-73

Verkman AS and Galietta LJV (2009). Chloride channels as drug targets. Nat Rev Drug Discov 8: 153-171 
Wang G (2010). State-dependent Regulation of Cystic Fibrosis Transmembrane Conductance Regulator (CFTR) Gating by a High Affinity Fe3+ Bridge between the Regulatory Domain and Cytoplasmic Loop 3. J Biol Chem 285: 40438-40447 Wang W, El Hiani Y and Linsdell P (2011). Alignment of transmembrane regions in the cystic fibrosis transmembrane conductance regulator chloride channel pore. J Gen Physiol 138: 165-178

Wang W, et al. (2010). ATP-independent CFTR channel gating and allosteric modulation by phosphorylation. Proc Natl Acad Sci USA 107: 3888-3893

Zhang L, et al. (2009). Architecture of the cystic fibrosis transmembrane conductance regulator protein and structural changes associated with phosphorylation and nucleotide binding. J Struct Biol. 167: 242-251 
Figure 1
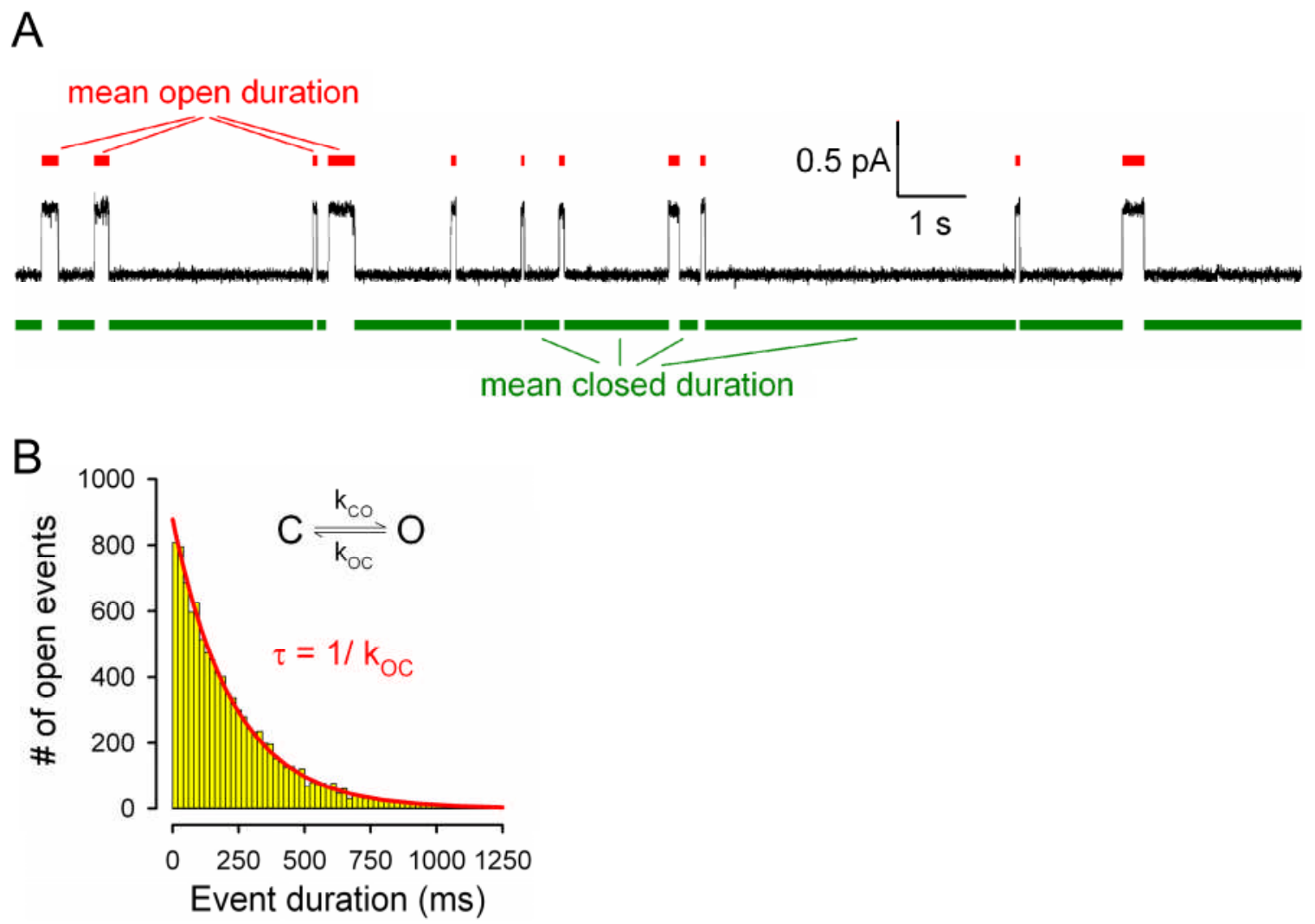

Figure 2

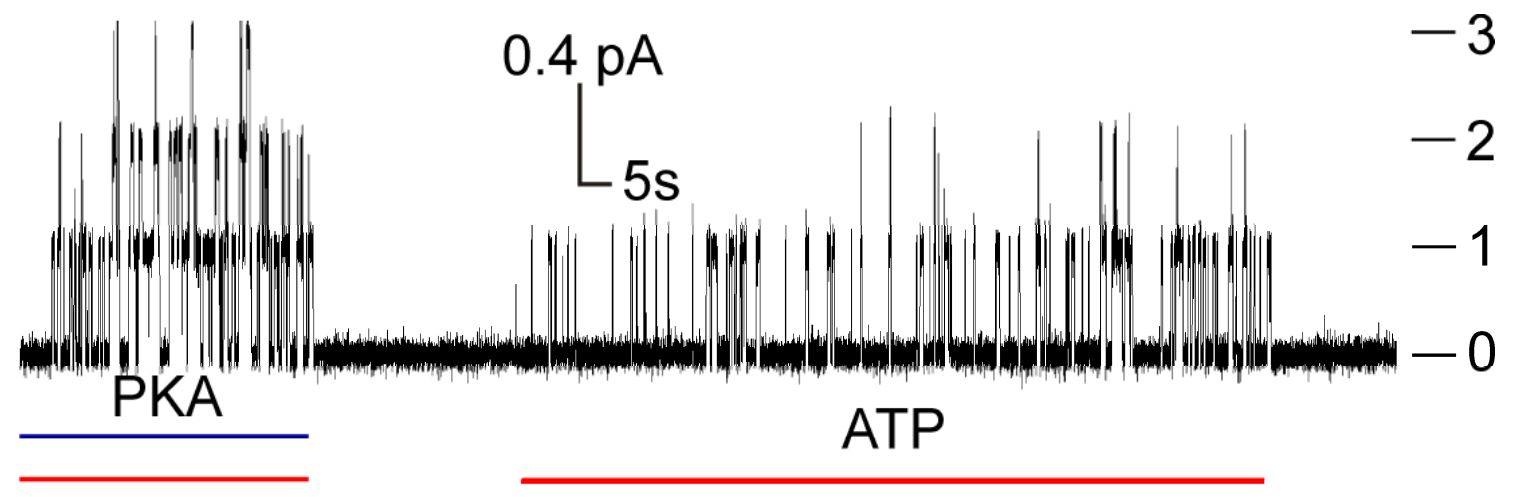


Figure 3

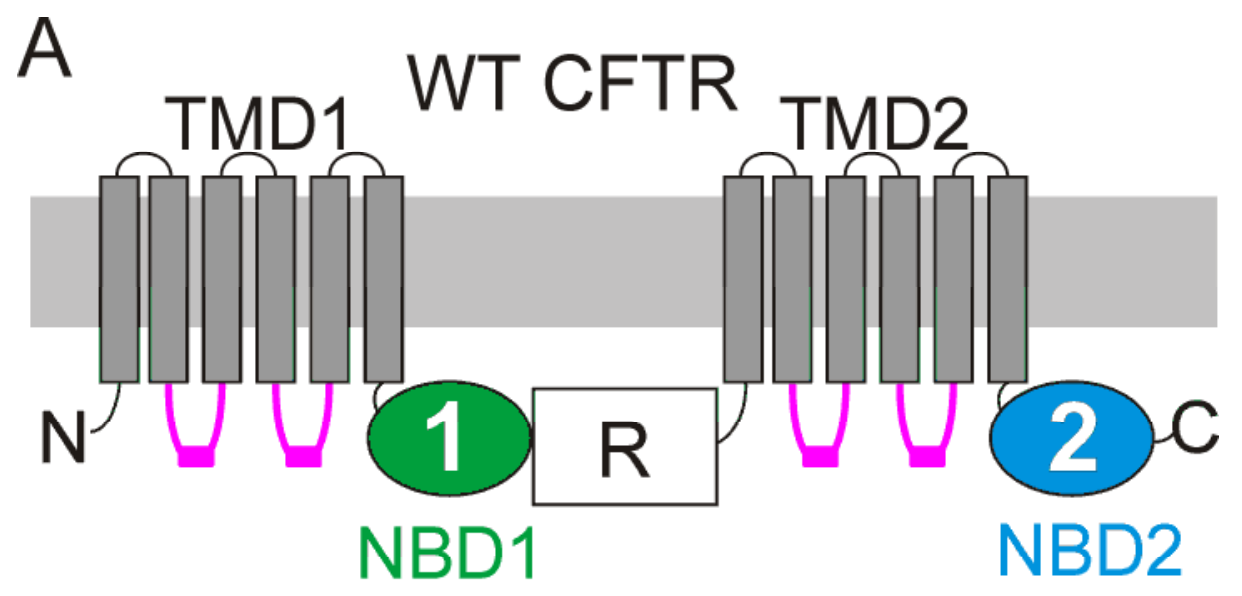

B

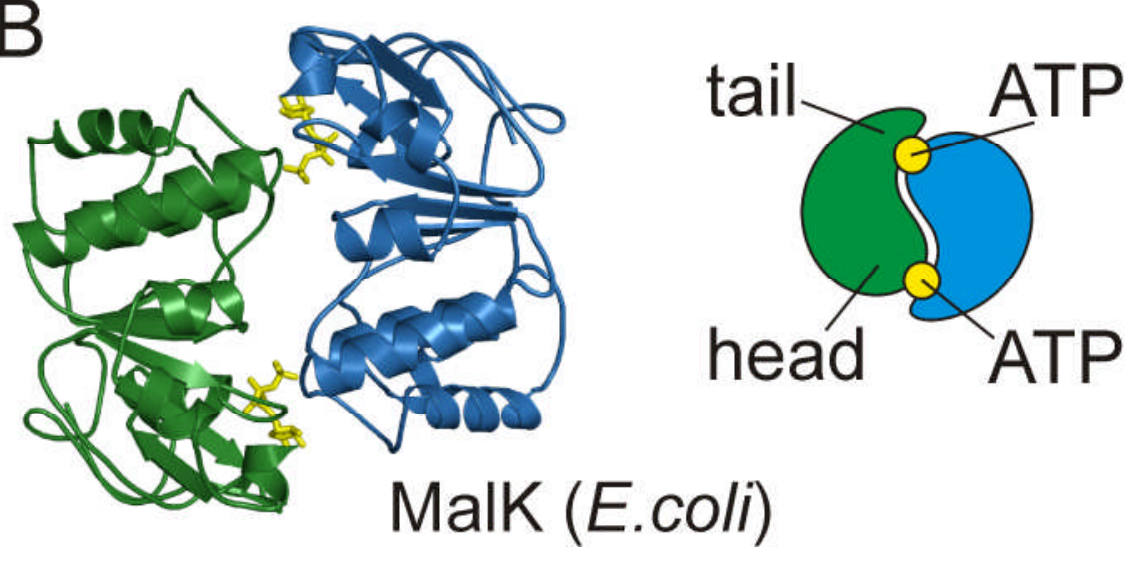


Figure 4

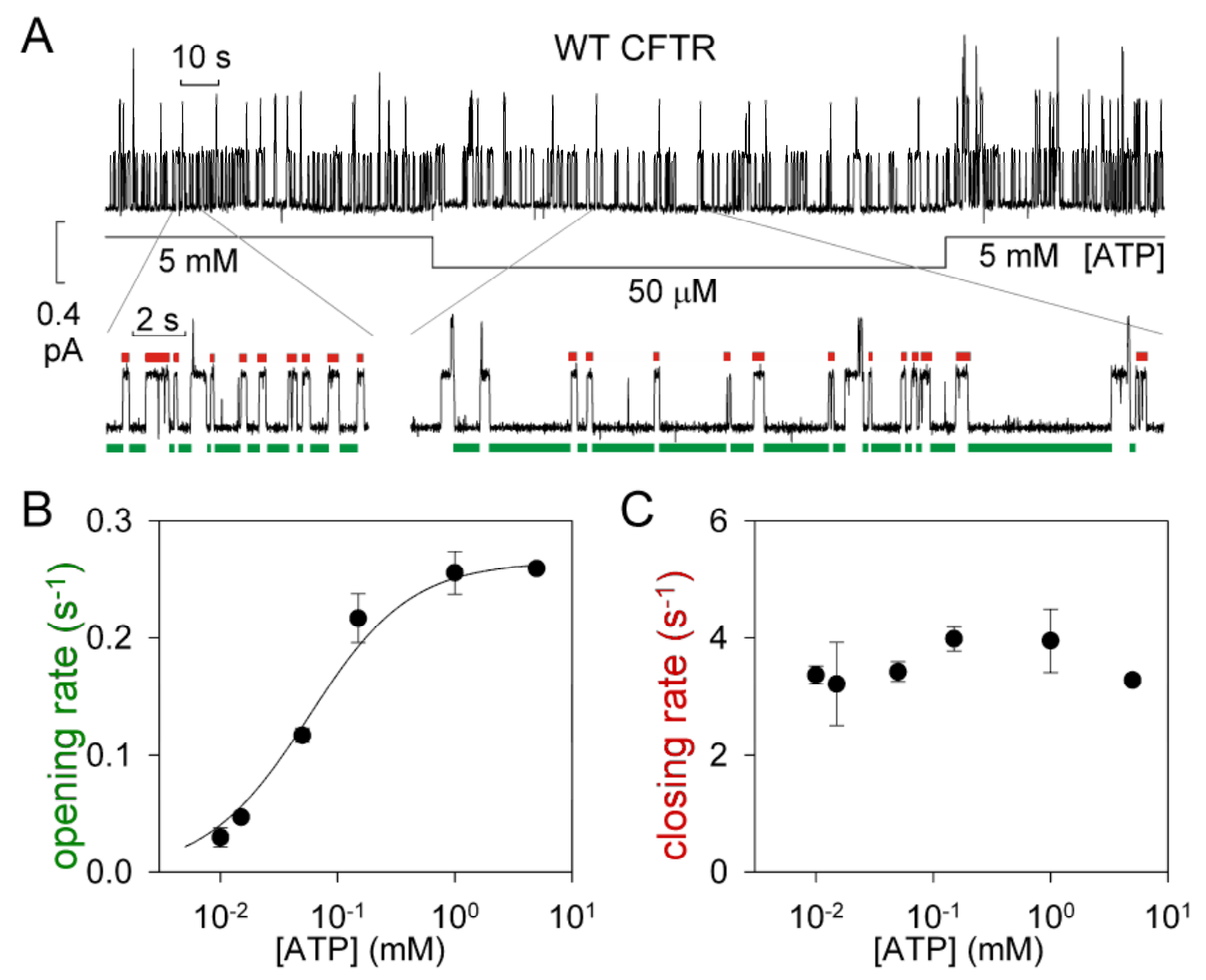


Figure 5

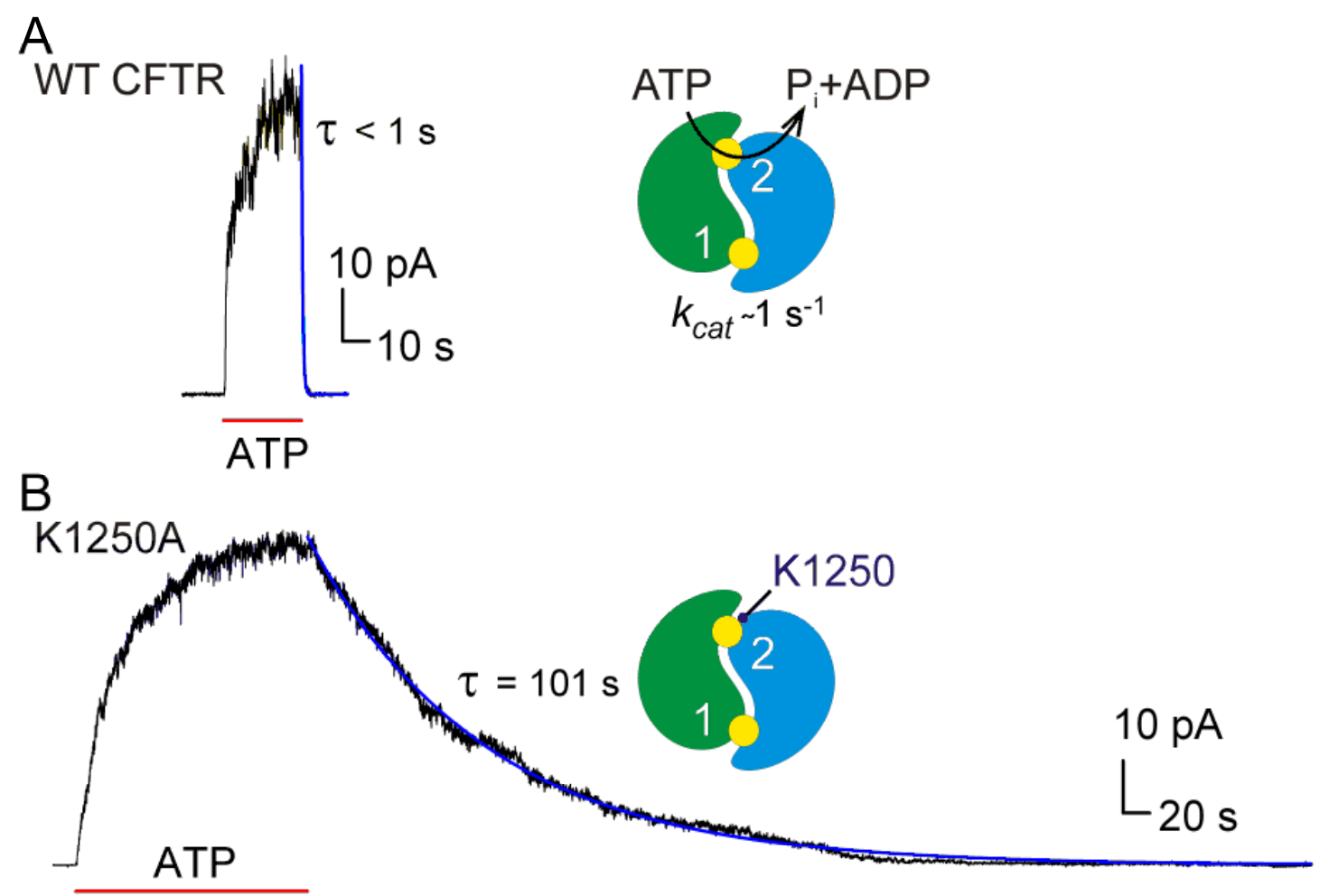


Figure 6

A

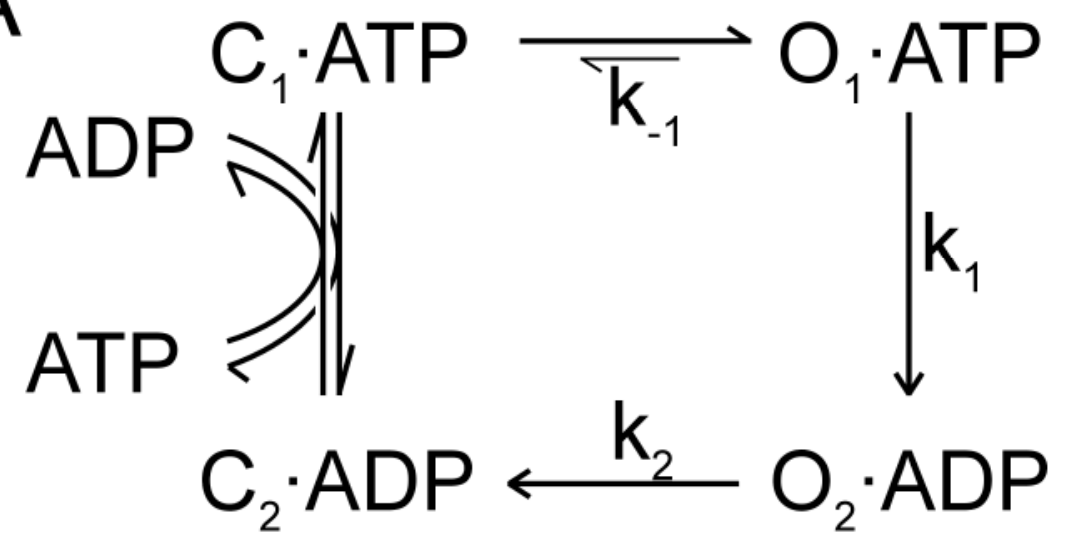

B

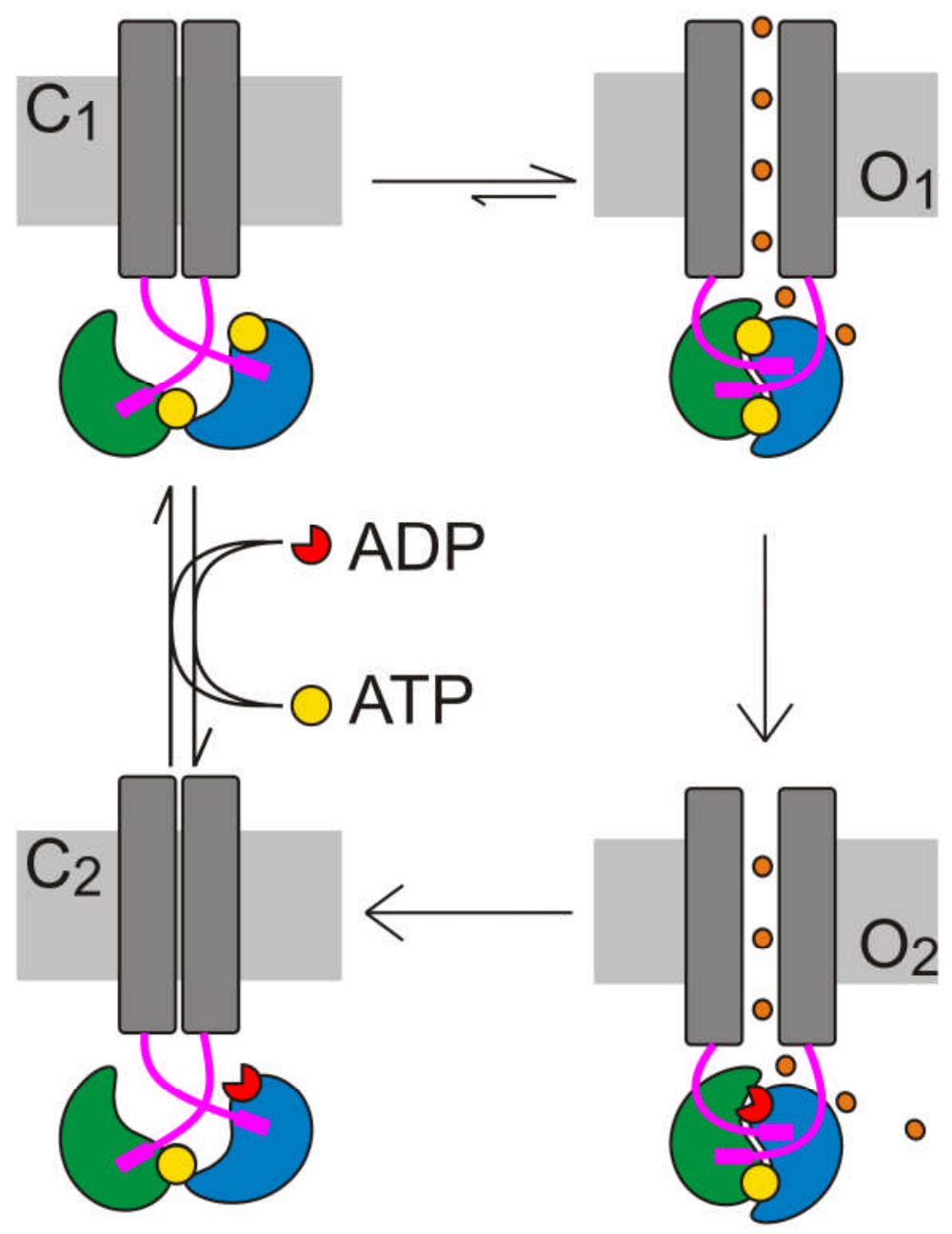


Figure 7
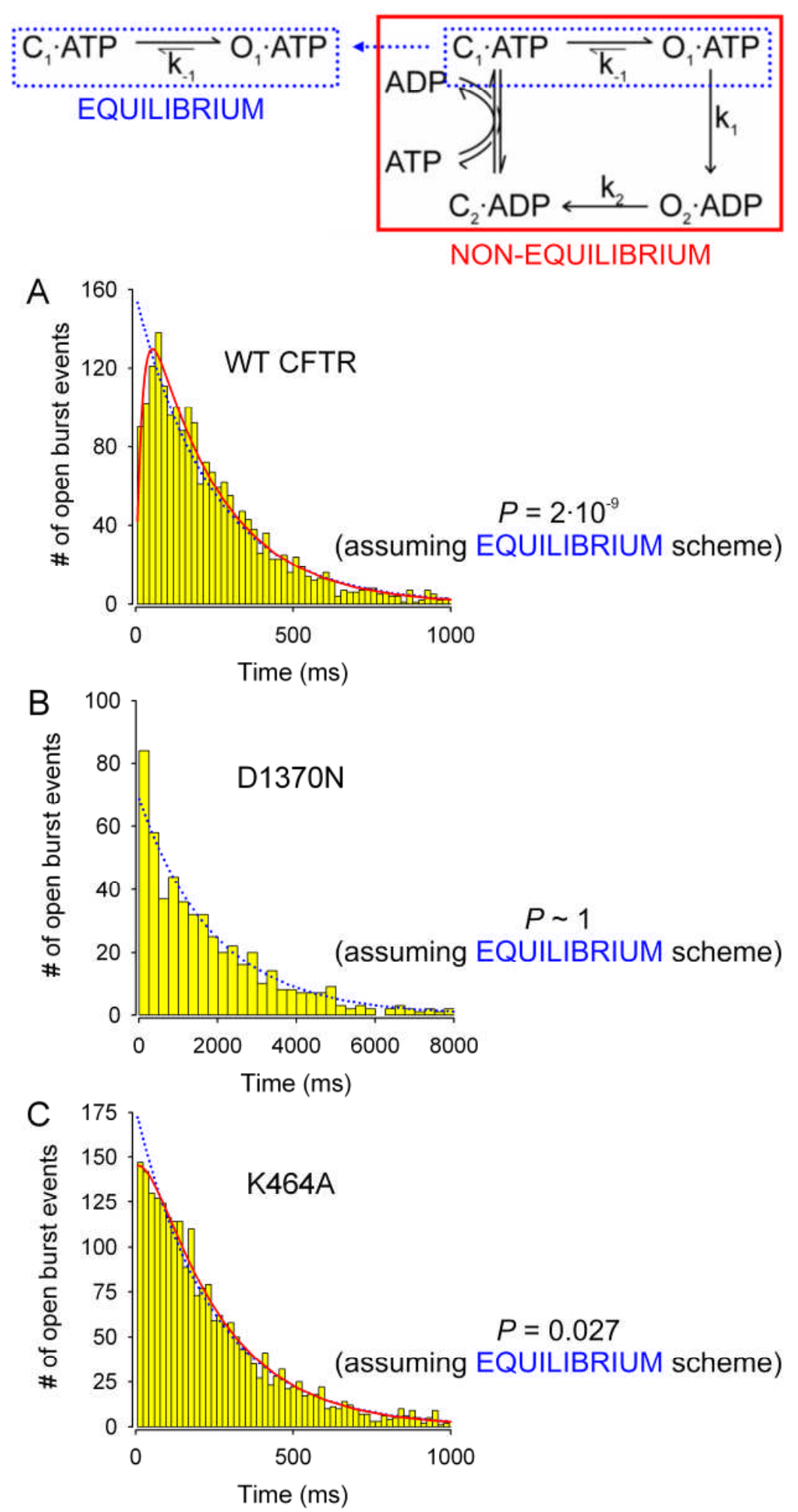
Figure 8.
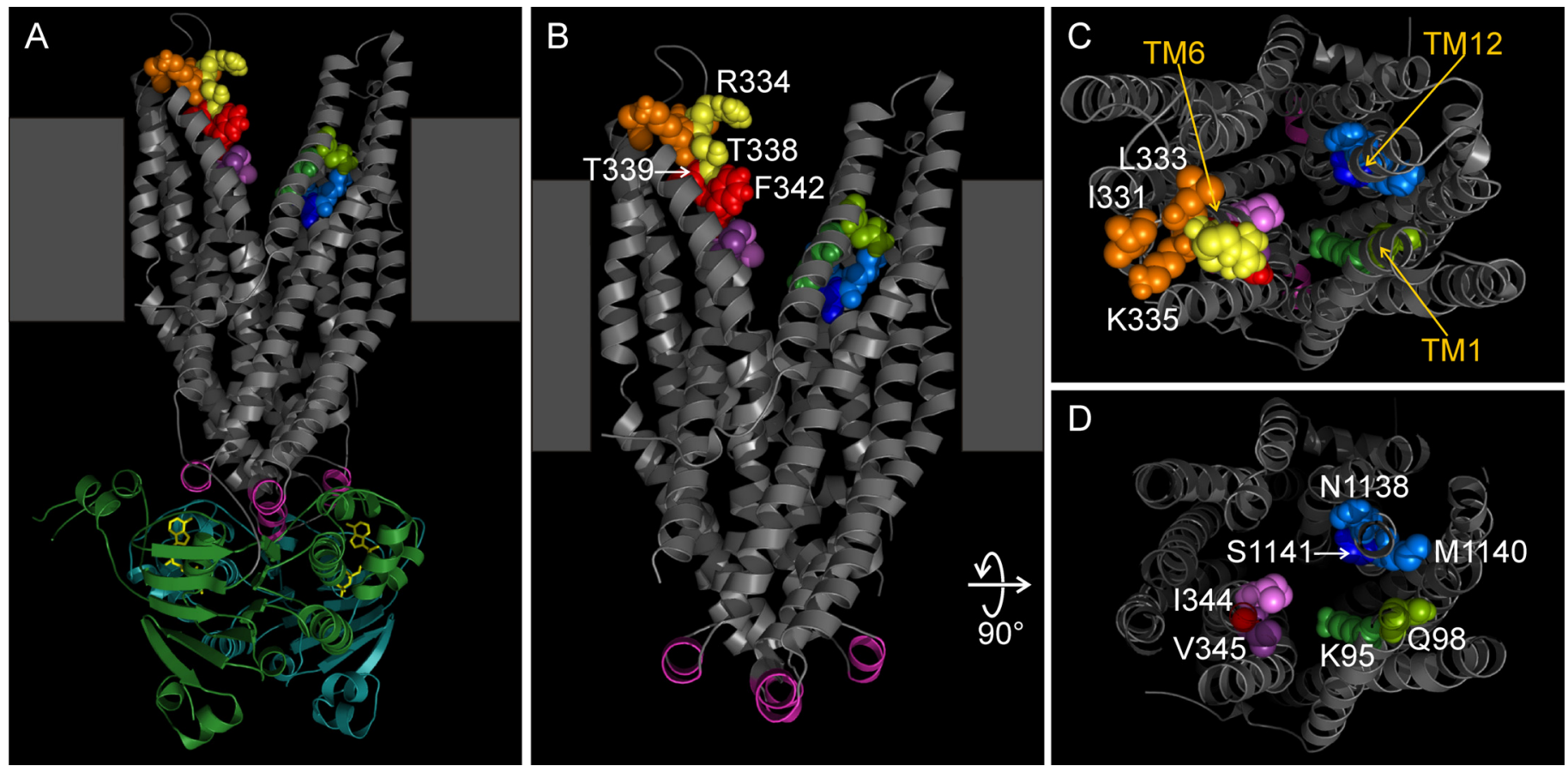